\title{
Checkpoint Inhibitor Relapsed Tumor
}

National Cancer Institute

\section{Source}

National Cancer Institute. Checkpoint Inhibitor Relapsed Tumor. NCI Thesaurus. Code C159787.

A finding indicating the treatment of a relapsed malignant tumor with checkpoint inhibitors to boost the immune response. 